\title{
The Association between Urinary Sodium Excretion and Metabolic Syndrome in Korean Adults from the 2010-2011 Korean National Health and Nutrition Examination Survey
}

\author{
Jeong Eun Seo', Hong Soo Lee', Sang Wha Lee', Kyung Won Shim', A Ri Byun', Jung Hwa Kim', Hee Jeong An', \\ Hyejin Chun ${ }^{2, *}$
}

'Department of Family Medicine, Ewha Womans University School of Medicine, Seoul, Korea

${ }^{2}$ Department of Family Medicine, Bundang CHA Medical Center, CHA University, Seongnam, Korea

Background: The sodium intake of Koreans was higher than that recommended by the World Health Organization. Urinary sodium, which is correlated with sodium intake, can be easily calculated by the Tanaka's equation. This study aimed to evaluate the association between urinary sodium and metabolic syndrome in Korean adults using the 2010-2011 Korean National Health and Nutrition Examination Survey (KNHANES).

Methods: A total of 5,870 participants from the 2010-2011 KNHANES were included in this study. Twenty-four hour urinary sodium was calculated by the Tanaka's equation using spot urine. Participants were divided into tertiles based on urinary sodium levels. The association between urinary sodium and metabolic syndrome was analyzed using multivariate logistic regression analysis.

Results: The odds ratios (ORs) and 95\% confidence intervals (CIs) of metabolic syndrome for the 2nd and 3rd tertile of urinary sodium levels was 1.51 (1.16-1.97) and 1.56 (1.23-1.97) compared to the lowest tertile of urinary sodium in men. The ORs and 95\% CIs of metabolic syndrome in women were $1.20(0.95-1.51)$ for the 2nd tertile and 2.16 (1.68-2.78) for the 3rd tertile. These associations remained statistically significant, even after adjusting for multiple covariates such as age, education, regular exercise, smoking, and alcohol consumption.

Conclusion: These findings indicate that urinary sodium is significantly associated with metabolic syndrome in Korean adults.

Keywords: Urine; Sodium; Metabolic Syndrome; Korea 


\section{INTRODUCTION}

High sodium intake has been reported to be related with high blood pressure (BP) and the risk of cardiovascular disease. ${ }^{1)}$ In addition, high sodium intake has been associated with type 2 diabetes mellitus and increased insulin resistance through hemodynamic changes in the kidneys by sustaining renal tubular reabsorption of sodium. ${ }^{2}$ Also, previous studies have demonstrated that high sodium intake is correlated with increased body weight and body fat mass. ${ }^{3,4)}$ According to the guidelines of the World Health Organization (WHO), sodium intake is recommended to be less than 2,000 $\mathrm{mg} / \mathrm{d}$, equal to $5 \mathrm{~g} / \mathrm{d}$ of salt. ${ }^{5)}$ Moreover, the Dietary Approaches to Stop Hypertension and American Heart Association recommend adjusting dietary sodium to 1,500 mg/d for cardiovascular health. ${ }^{6}$ However, the 2009 Korea National Health Statistics reported the average sodium intake of Koreans was 4,600 mg/d (men 5,381 mg/d and women 3,813 mg/d). ${ }^{7}$ These levels are more than two times higher than those recommended by the WHO. The high sodium intake of Koreans might be due to the traditional dietary habits of Korea. Most Koreans eat kimchi, soup, and stew at almost every meal. Kimchi is made with cabbage that is mainly fermented by salt, a salted fish source, and red pepper. In addition, most of the soup and stew are seasoned with salt or soy sauce. ${ }^{8)}$ Therefore, Koreans have no choice but to consume a large amount of salt.

Metabolic syndrome, a cluster of risk factors including abdominal obesity, high BP, high fasting glucose, high triglycerides (TG), and low high-density lipoprotein cholesterol (HDL cholesterol), increases the risk of cardiovascular disease or type 2 diabetes mellitus. ${ }^{9,10)}$ The prevalence of metabolic syndrome among participants in the Korean National Health and nutrition Examination Surveys (KNHANES) from 1998 to 2007 has increased significantly from $24.9 \%$ to $31.3 \%{ }^{11)}$ Some studies have identified a relationship between sodium intake and metabolic syndrome. ${ }^{12-17)}$ They showed that daily sodium intake was higher in subjects with metabolic syndrome than those without metabolic syndrome. ${ }^{12-17)}$ Therefore, Koreans especially might be concerned about this increase in people with metabolic syndrome due to their high sodium intake.

There are two methods to estimate salt intake: (1) a dietary survey in which one recalls the foods consumed over the previous 24 hours using a questionnaire and (2) measurement of 24-hour urinary sodium excretion. ${ }^{12-14,18)}$ The latter is preferred because the dietary survey relies on individual memories and is vulnerable to recall bias. However, it is not easy to collect 24-hour urine samples for all examiners. Therefore, many researchers calculate 24-hour urine sodium by using several equations from the spot urine. ${ }^{19)}$ The most commonly used equation is Tanaka's equation. The sodium levels from Tanaka's equation using the spot urine have a significant correlation with the sodium levels measured by 24-hour urine sample. ${ }^{20,21)}$

There have been a few studies done to evaluate the association between sodium excretion or sodium intake and metabolic syndrome. ${ }^{12-17)}$ To the best of our knowledge, no previous study has evaluated the relationship between estimated 24-hour urinary sodium ex- cretion using spot urine and metabolic syndrome in representatives from Korea. Therefore, in this study, we aimed to investigate the association between urinary sodium and metabolic syndrome in Koreans by spot urine.

\section{METHODS}

\section{Study Populations and Data Collection}

This study was a cross-sectional study to examine the association between urinary sodium and prevalence of metabolic syndrome in Korean adults participating in the KNHANES. All data were collected from 2010-2011 of the KNHANES, which was a nationally representative survey conducted by the Korea Centre for Disease Control and Prevention. Among the 6,084 participants who were 20 or older, 214 participants with a medical history such as myocardial infarction, angina, or renal failure were excluded because these participants might take medications that have an effect on sodium excretion. Therefore, a total of 5,870 participants were included in this study. Informed consent was obtained from all participants before participating in KNHANES.

KNHANES was composed of a health interview survey, a nutrition survey including the frequency and kind of food intake, and a health examination survey including a physical examination, laboratory tests, and a radiologic study. The health interview survey included questions on age, gender, education, socioeconomic status, medical history, health behaviors, and quality of life. Nutrition was evaluated by administering a validated food frequency questionnaire (FFQ) to each participant. The FFQ requested information regarding consumed food items and the frequency of food intake, which were quantified using 9 categories: 'never or seldom,' 'once a month,' 'two to three times a month,' 'one to two times a week,' 'three to four times a week, 'five to six times a week,' 'once a day,' 'twice a day, and 'three or more times every day.' A health behavior survey examining such behaviors as smoking, alcohol consumption, and exercise was evaluated from a self-administered questionnaire. Alcohol consumption was investigated by questions about frequency of alcohol consumption on a weekly basis and the usual amount of alcohol consumption on a daily basis. Alcohol was defined as one or more times a month. In addition, smoking was defined as more than a lifetime 5 boxes (100 cigarettes) and participants who reported smoking were considered current smokers. Participants were asked about their physical activity on a weekly basis. Regular exercise was defined as exercising more than 20 minutes, enough to sweat at one time, and regularly three times a week or more.

Body mass index (BMI) was calculated as the weight ( $\mathrm{kg}$ ) divided by square of the height (m). Waist circumference (WC) was measured while standing at the mid-point between the lower border of last rib and the ridge of iliac bone of the mid-axillary line by a single examiner. BP was measured by a standardized mercury sphygmomanometer after at least 5 minutes of rest. Blood sampling was performed from the antecubital vein in the morning after at least 8 hours of fasting. TG, HDL cholesterol, and fasting blood glucose were measured using an 
enzymatic method. Urine sampling was performed from collecting intermediate urine. Urinary sodium levels were measured using the ionselective electrode method. Blood and urine laboratory values were measured with a Hitachi Automatic Analyzer 7600 (Hitachi HighTechnologies, Tokyo, Japan).

\section{Estimation of Sodium Intake by $24-$ Hour Urinary Sodium Excretion}

We estimated the 24-hour urinary sodium from the sodium and creatinine of spot urine samples using Tanaka's equation. ${ }^{18,20,21)}$ Tanaka's equation is as follows:

Estimated 24-hour urinary sodium excretion $(\mathrm{mmol} / \mathrm{d})=21.98 \times$ $\mathrm{XNA}^{0.392}, \mathrm{XNA}=\{$ spot urine sodium $(\mathrm{mmol} / \mathrm{L}) /[$ spot urine creatinine $(\mathrm{mg} / \mathrm{dL}) \times 10]\} \times$ estimated 24-hour urinary creatinine $(\mathrm{mg} / \mathrm{d})$, estimated 24 -hour urine creatinine $(\mathrm{mg} / \mathrm{d})=-2.04 \times$ age (years)+14.89×body weight $(\mathrm{kg})+16.14 \times$ height $(\mathrm{cm})-2,244.45$

\section{Definition of Metabolic Syndrome}

Metabolic syndrome was defined according to the National Cholesterol Education Program, Adult Treatment Panel III. ${ }^{22,23)}$ Central obesity among the components of metabolic syndrome was defined according to guidelines of the Korean Society for the Study of Obesity. ${ }^{24)}$ Metabolic syndrome was diagnosed if a subject has three or more of the following criteria ${ }^{10,22,23}$ : (1) central obesity (WC $\geq 90 \mathrm{~cm}$ for men and $\geq 85 \mathrm{~cm}$ for women), (2) elevated TG (fasting TG $\geq 150 \mathrm{mg} / \mathrm{dL}$ ), (3) reduced high-density lipoprotein cholesterol (fasting HDL cholesterol $<40 \mathrm{mg} / \mathrm{dL}$ in men and $<50 \mathrm{mg} / \mathrm{dL}$ in women), (4) elevated BP (systolic $\mathrm{BP} \geq 130 \mathrm{~mm} \mathrm{Hg}$ and/or diastolic $\mathrm{BP} \geq 85 \mathrm{~mm} \mathrm{Hg}$, or use of anti-hypertensive medications), and (5) elevated fasting glucose (fasting glucose $>100 \mathrm{mg} / \mathrm{dL}$ or use of anti-diabetic medications or insulin).

\section{Statistical Analyses}

The KNHANES had a complex sample design and was analyzed by multi-stage stratification and weighted. Data in the text and tables are expressed as the mean and standard error for continuous variables and as the sample size or counts with percentage for categorical variables. All analyses were performed according to gender. A t-test for continuous variables and a chi-square test for non-continuous variables were used to analyze the statistical differences between groups in accordance to the presence or absence of metabolic syndrome. A simple linear regression was used to analyze the trend of mean urinary sodium according to the number of the components of metabolic syndrome. A one-way analysis of variance with a Dunnett's post-hoc test was used to compare the mean urinary sodium among 5 groups according to the number of the components of metabolic syndrome. Participants were divided into gender-specific tertiles on the basis of urinary sodium level as follows: tertile 1, 24-hour urinary sodium $<130.25 \mathrm{mmol} / \mathrm{d}$; tertile 2, 24-hour urinary sodium 130.25 to 160.79 $\mathrm{mmol} / \mathrm{d}$; and tertile $3, \geq 160.79 \mathrm{mmol} / \mathrm{d}$ in men, and tertile 1,24 -hour urinary sodium $<128.28 \mathrm{mmol} / \mathrm{d}$; tertile 2 , 24-hour urinary sodium 128.28 to $157.95 \mathrm{mmol} / \mathrm{d}$; and tertile $3, \geq 157.95 \mathrm{mmol} / \mathrm{d}$ in women. A linear-by-linear association was used to analyze the differences in prevalence of metabolic syndrome by 24 -hour urinary sodium with $\mathrm{P}$ for the trend. Compared to the lowest tertile of 24-hour urinary sodium, the odds ratios (ORs) of metabolic syndrome in the highest 2 tertile groups was calculated by logistic regression. Additionally, logistic regression was used to analyze the independent risk for metabolic syndrome according to 24-hour urinary sodium after adjusting for clinical variables related to metabolic syndrome such as age, education, regular exercise, smoking, and alcohol consumption. P-values less than 0.05 were considered to be statistically significant. Statistical analyses were performed PASW SPSS Statistics ver. 18.0 (SPSS Inc., Chicago, IL, USA).

Table 1. Characteristics of the study group according to MS status

\begin{tabular}{|c|c|c|c|c|c|c|}
\hline \multirow{2}{*}{ Variable } & \multicolumn{3}{|l|}{ Men } & \multicolumn{3}{|l|}{ Women } \\
\hline & MS & No MS & P-value* & MS & No MS & P-value* \\
\hline Participants & 910 & 1,710 & & 1,003 & 2,247 & \\
\hline Age (y) & $49.7 \pm 0.5$ & $42.0 \pm 0.6$ & $<0.01$ & $59.5 \pm 0.6$ & $43.4 \pm 0.5$ & $<0.01$ \\
\hline Body mass index $\left(\mathrm{kg} / \mathrm{m}^{2}\right)$ & $26.6 \pm 0.1$ & $23.4 \pm 0.1$ & $<0.01$ & $26.5 \pm 0.1$ & $22.6 \pm 0.1$ & $<0.01$ \\
\hline Waist circumference (cm) & $92.6 \pm 0.4$ & $81.8 \pm 0.3$ & $<0.01$ & $89.2 \pm 0.3$ & $75.5 \pm 0.3$ & $<0.01$ \\
\hline High school graduates (\%) & $586(69.4)$ & $1,273(81.1)$ & $<0.01$ & $242(26.2)$ & $1,522(71.1)$ & $<0.01$ \\
\hline Smoking $(\%)$ & $380(48.6)$ & $749(48.2)$ & 0.89 & $44(6.2)$ & $104(5.7)$ & 0.74 \\
\hline Alcohol (\%) & $696(80.4)$ & $1,289(77.8)$ & 0.18 & $249(28.5)$ & $900(43.6)$ & $<0.01$ \\
\hline Regular exercise (\%) & $192(22.3)$ & $418(26.6)$ & 0.06 & $180(17.6)$ & $456(21.0)$ & 0.04 \\
\hline Systolic blood pressure (mm Hg) & $129.0 \pm 0.8$ & $115.4 \pm 0.5$ & $<0.01$ & $132.4 \pm 0.8$ & $111.4 \pm 0.5$ & $<0.01$ \\
\hline Diastolic blood pressure $(\mathrm{mm} \mathrm{Hg})$ & $83.8 \pm 0.5$ & $75.2 \pm 0.4$ & $<0.01$ & $78.5 \pm 0.5$ & $71.1 \pm 0.3$ & $<0.01$ \\
\hline Fasting plasma glucose (mg/dL) & $113.7 \pm 1.1$ & $94.7 \pm 0.7$ & $<0.01$ & $112.7 \pm 1.2$ & $90.7 \pm 0.3$ & $<0.01$ \\
\hline Triglyceride (mg/dL) & $270.8 \pm 9.8$ & $128.8 \pm 3.7$ & $<0.01$ & $202.2 \pm 4.5$ & $92.3 \pm 1.3$ & $<0.01$ \\
\hline High-density lipoprotein cholesterol (mg/dL) & $42.1 \pm 0.4$ & $51.9 \pm 0.4$ & $<0.01$ & $44.8 \pm 0.4$ & $58.2 \pm 0.3$ & $<0.01$ \\
\hline Urinary sodium $(\mathrm{mmol} / \mathrm{d})$ & $150.5 \pm 1.8$ & $142.7 \pm 1.1$ & $<0.01$ & $152.3 \pm 1.7$ & $139.3 \pm 1.0$ & $<0.01$ \\
\hline
\end{tabular}

Values are presented as number, means \pm standard error, or number (\%), unless otherwise stated.

${ }^{\star}$ Calculated by t-test (continuous variables) or chi-square test (categorical variables). 


\section{RESULTS}

\section{Characteristics of the Group according to Metabolic Syndrome}

The characteristics of the groups in accordance with metabolic syndrome status are presented according to gender in Table 1. A total of 5,870 subjects (2,620 men [44.6\%] and 3,250 women [55.4\%]) participated in this study. Among them, 910 (34.7\%) men and 1,003 (30.9\%) women were confirmed as having metabolic syndrome. Participants with metabolic syndrome were older, had a higher BMI and WC, and a lower HDL cholesterol and high school education than participants without metabolic syndrome in both men and women (all $\mathrm{P}<0.01$ ). However, alcohol consumption and regular exercise were more prevalent in participants without metabolic syndrome in only women. Systolic BP, diastolic BP, fasting glucose, and TG showed a significant difference in both men and women; these levels were significantly higher in the group with metabolic syndrome than in the group without metabolic syndrome. Urinary sodium was significantly higher in the group with metabolic syndrome than those who did not have metabolic syndrome $(\mathrm{P}<0.01)$. There was no significant difference in smoking.

\section{Urinary Sodium according to the Number of Metabolic Syndrome Components}

The mean 24-hour urinary sodium according to the number of metabolic syndrome components is described in Figure 1. There was a significant increasing trend of mean urinary sodium in men and women (both $\mathrm{P}$ for trend $<0.01$ ) according to the number of metabolic syndrome components.

\section{Association between Urinary Sodium and Metabolic Syndrome}

Multivariate logistic regression analysis was conducted to examine the relationship between urinary sodium and metabolic syndrome (Table 2). ORs (95\% confidence intervals [CI]) for metabolic syndrome in the 2nd tertile group and the 3rd tertile group in men were 1.51 (1.16-1.97) and 1.56 (1.23-1.97) compared to the lowest tertile group, respectively ( $\mathrm{P}$ for trend $<0.01)$. In women, ORs (95\% CI) for metabolic syndrome in the 2nd and the 3rd tertile groups were 1.20 (0.95-1.51) and 2.16 (1.68-2.78), respectively ( $\mathrm{P}$ for trend $<0.01$ ). When age was adjusted in men and women, these associations still remained statistically significant ( $\mathrm{P}$ for trend=0.01 in men, $\mathrm{P}$ for trend $<0.01$ in women). Furthermore, even after adjusting for multiple covariates such as age, education, regular exercise, smoking, and alcohol consumption, these associations were also statistically significant. In men, multivariate adjusted ORs for metabolic syndrome in the 2nd and the 3rd tertile groups were 1.42 (1.08-1.85) and 1.40 (1.10-1.78), respectively (P for trend $<0.01)$. In women, multivariate adjusted ORs for metabolic syndrome of the 2nd and the 3rd tertile groups were 1.03 (0.80-1.32) and 1.54 (1.18-2.02), respectively ( $P$ for trend $<0.01$ ). Comparing the ORs of individual components of the metabolic syndrome, central obesity, elevated TG, and elevated BP or medication had a significantly positive relationship in men even after multivariate adjustment. On the other hand, central obesity, elevated TG, and low HDL cholesterol were more associated with 24-hour urinary sodium in women (all $\mathrm{P}$ for trend $<0.01)$. Furthermore, these associations still remained significant even after age and multivariate adjustments. Table 3 shows how much the prevalence of metabolic syndrome increases according to each 100 $\mathrm{mmol} / \mathrm{L}$ increase in urinary sodium. When urinary sodium excretion is increased by $100 \mathrm{mmol}$, the ORs of metabolic syndrome increases by 1.79 times in men and 2.64 times in women, respectively (both Pvalue $<0.01$ ). After multivariate adjustment, this association still remained.

\section{DISCUSSION}

A

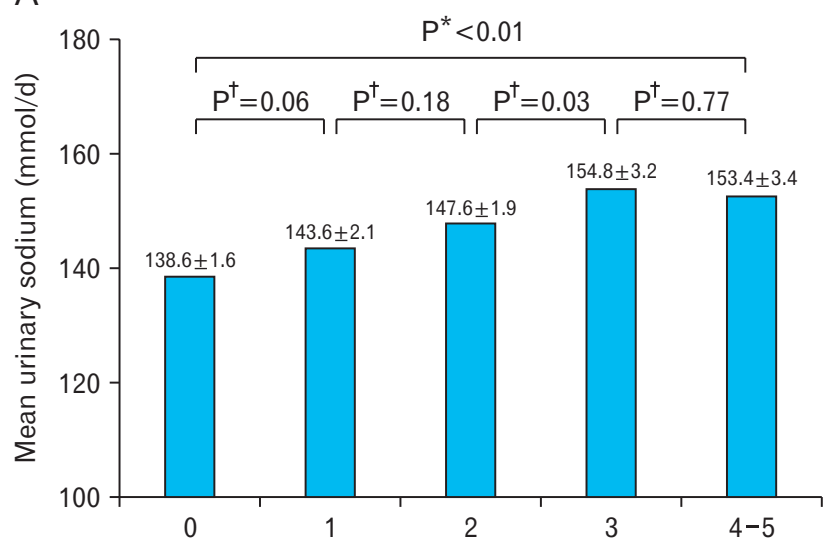

No. of metabolic syndrome component
B

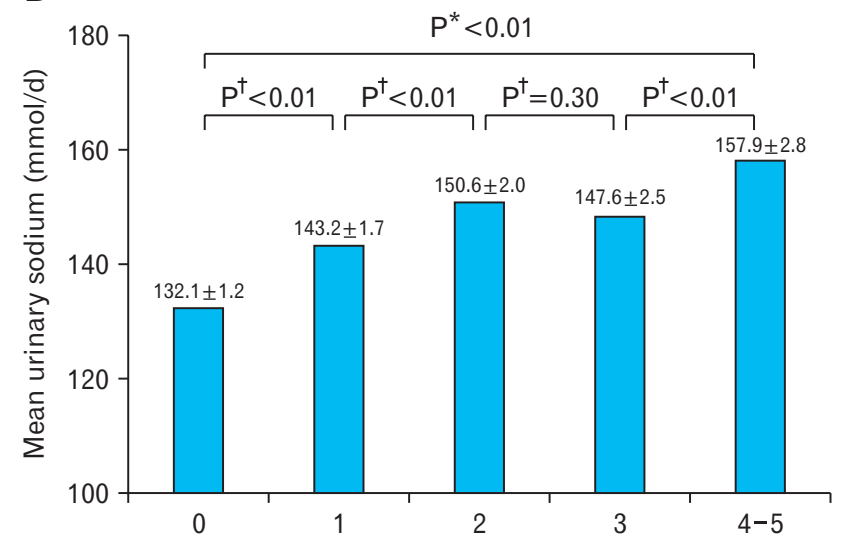

No. of metabolic syndrome component

Figure 1. Mean urinary sodium according to the number of metabolic syndrome risk factors. (A) Men. (B) Women. Values are presented as mean \pm standard error. ${ }^{*}$-values are calculated using simple linear regression. 'P-values are calculated using one-way analysis of variance with post-hoc Dunnett's test. 


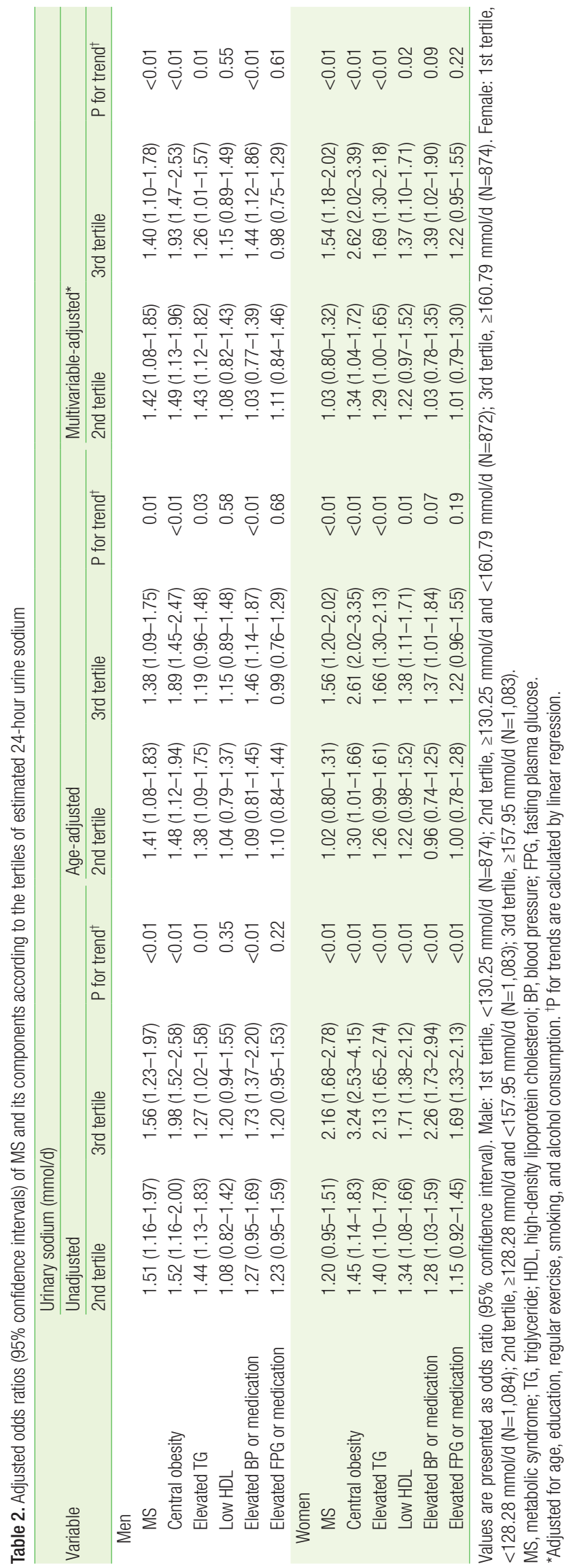

In this study, we evaluated the risk of metabolic syndrome according to urinary sodium in Korean adults. The result of this study showed a significant association between urinary sodium and metabolic syndrome in Korean adults. We could confirm that participants with metabolic syndrome had higher urinary sodium than those without metabolic syndrome. Moreover, mean urinary sodium tended to increase according to the number of metabolic syndrome components in both men and women (both P-values $<0.01$ ). Compared to the lowest tertile group of urinary sodium levels, subjects in the higher tertile groups had a higher risk of metabolic syndrome. These results suggest that there is a significant association between urinary sodium and metabolic syndrome. In addition, sodium intake might be a potential risk factor for metabolic syndrome because 24-hour urinary sodium has a close correlation with sodium intake. Moreover, 24-hour urinary sodium can be easily calculated using Tanaka's equation from a spot urine sample. Therefore, the risk of metabolic syndrome can be easily predicted by the spot urine test. We also found that there was a difference in the components of metabolic syndrome associated with urinary sodium according to gender. The mechanism of difference according to gender was unclear. However, we might explain this as being caused by gender-specific body composition and characteristics. In this study, the prevalence of metabolic syndrome and obesity markers such as BMI and WC were higher in men than in women. Previous studies showed that the prevalence of sodium sensitivity was high in individuals with metabolic syndrome and obesity was a risk factor for BP elevation. ${ }^{16)}$ Therefore, elevated BP among the components of metabolic syndrome might be meaningful in only men. In addition, high sodium intake tended to increase overall glucocorticoid production, which resulted in increased fat mass and decreased muscle mass. Therefore, a high sodium diet had an influence on gender-specific metabolic profiles and body composition. There is also a possibility that these mechanisms had an influence on the gender-specific differences in the association between urinary sodium and each component of metabolic syndrome. ${ }^{18)}$

There are several mechanisms explaining how urinary sodium could contribute to metabolic syndrome. First, high sodium intake is associated with an increased risk of obesity. ${ }^{16,25-27)}$ Although the mechanism of how sodium intake is directly related to obesity remains unclear, increased food intake might increase the sodium intake and sugar-sweetened beverages accompanied by food intake have a high sodium content. ${ }^{25,26)}$ For this reason, sodium intake is likely to be related to an unfavorable metabolic profile. Second, hemodynamic changes in the kidney due to increasing sodium intake can induce an increase in BP, one of the criteria of metabolic syndrome. ${ }^{28)}$ Although the degree of sensitivity to sodium intake differs among people, excess sodium intake leads to sodium retention, increases in cardiac output, and results in increased vascular resistance. Moreover, if sodium intake is increased, the mineralocorticoid receptor is activated and distal sodium reabsorption is increased. In turn, these changes stimulate alphal and betal adrenoceptor, leading to the development of salt-induced hypertension. It has also been reported that a high sodium diet increases 
Table 3. Adjusted ORs of MS and its components associated with a $100 \mathrm{mmol}$ increase in urinary sodium excretion

\begin{tabular}{|c|c|c|c|c|c|c|}
\hline \multirow{2}{*}{ Variable } & \multicolumn{2}{|l|}{ Unadjusted } & \multicolumn{2}{|l|}{ Age-adjusted } & \multicolumn{2}{|l|}{ Multivariable-adjusted* } \\
\hline & OR (95\% Cl) & P-value & OR (95\% Cl) & P-value & OR $(95 \% \mathrm{Cl})$ & $P$-value \\
\hline \multicolumn{7}{|l|}{ Men } \\
\hline MS & $1.79(1.37-2.36)$ & $<0.01$ & $1.58(1.20-2.08)$ & $<0.01$ & $1.60(1.21-2.12)$ & $<0.01$ \\
\hline Central obesity & $2.08(1.54-2.83)$ & $<0.01$ & $1.98(1.46-2.68)$ & $<0.01$ & $2.02(1.49-2.75)$ & $<0.01$ \\
\hline Elevated TG & $1.34(1.04-1.72)$ & 0.02 & $1.24(0.96-1.60)$ & 0.09 & $1.32(1.02-1.71)$ & 0.03 \\
\hline Low HDL & $1.19(0.88-1.61)$ & 0.26 & $1.12(0.83-1.52)$ & 0.46 & $1.12(0.83-1.53)$ & 0.46 \\
\hline Elevated BP or medication & $2.12(1.59-2.84)$ & $<0.01$ & $1.78(1.30-2.42)$ & $<0.01$ & $1.78(1.29-2.45)$ & $<0.01$ \\
\hline Elevated FPG or medication & $1.25(0.96-1.64)$ & 0.10 & $1.08(0.75-1.36)$ & 0.96 & $1.00(0.74-1.35)$ & 1.00 \\
\hline \multicolumn{7}{|l|}{ Women } \\
\hline MS & $2.64(1.98-3.53)$ & $<0.01$ & $1.81(1.33-2.48)$ & $<0.01$ & $1.77(1.28-2.45)$ & $<0.01$ \\
\hline Central obesity & $4.56(3.46-6.01)$ & $<0.01$ & $3.54(2.64-4.74)$ & $<0.01$ & $3.47(2.56-4.70)$ & $<0.01$ \\
\hline Elevated TG & $2.31(1.74-3.06)$ & $<0.01$ & $1.70(1.27-2.28)$ & $<0.01$ & $1.72(1.26-2.35)$ & $<0.01$ \\
\hline Low HDL & $1.77(1.40-2.25)$ & $<0.01$ & $1.36(1.08-1.73)$ & 0.01 & $1.37(1.07-1.75)$ & 0.01 \\
\hline Elevated BP or medication & $2.70(1.95-3.75)$ & $<0.01$ & $1.47(1.02-2.14)$ & 0.04 & $1.45(0.99-2.12)$ & 0.06 \\
\hline Elevated FPG or medication & $1.88(1.46-2.44)$ & $<0.01$ & $1.29(0.99-1.67)$ & 0.06 & $1.27(0.97-1.65)$ & 0.08 \\
\hline
\end{tabular}

$\mathrm{OR}$, odds ratio; $\mathrm{MS}$, metabolic syndrome; Cl, confidence intervals; TG, triglyceride; HDL, high-density lipoprotein cholesterol; BP, blood pressure; FPG, fasting plasma glucose. *Adjusted for age, education, regular exercise, smoking, and alcohol consumption.

glucocorticoid production. ${ }^{29)}$ Mineralocorticoid receptor activation and glucocorticoid production were correlated with an unfavorable metabolic profile such as elevation of BP, insulin resistance, and dyslipidemia. ${ }^{14)}$ Also, a high sodium diet is likely related to stimulation of adipocyte hypertrophy by increasing leptin and lipogenic enzymatic activity. ${ }^{3,30)}$ Accordingly, it can accumulate fat and cause obesity and dyslipidemia.

Several studies have reported an association between sodium intake and metabolic syndrome. One study of a Chinese population reported that sodium intake was a dose-response relationship with central obesity, hypertension, and elevated TG. ${ }^{12)}$ Another study of a Spanish population reported that sodium intake was related with obesity and high $\mathrm{BP}^{15)}$ and a study of Finns reported that sodium intake was related with BMI, fasting glucose, and BP. ${ }^{17)}$ In addition, according to the DONALD (Dortmund Nutritional and Anthropometric Longitudinally Designed) study, high sodium intake is associated with urinary sodium excretion and children with a high urinary sodium excretion tended to have an increase in body fat. ${ }^{4)}$ Based on these study results, it has been hypothesized that sodium intake or urinary sodium excretion may contribute to unfavorable metabolic risk profiles in spite of ethnic differences. There was also one Korean study in which 463 Koreans showed a positive association between sodium intake and metabolic syndrome. ${ }^{16)}$ However, our study is more significant in that we demonstrated a positive association between urinary sodium and metabolic syndrome using KNHANES data, which represent Koreans, and we therefore minimized the recall bias that can occur from the dietary survey.

Nevertheless, some limitations should be considered when interpreting our results. First, this study has a limitation as it is a cross-sectional study and a causal relationship between urinary sodium and prevalence of metabolic syndrome cannot be determined. Therefore, the pathophysiological effect of urinary sodium on predisposition to metabolic syndrome should be investigated further. Although the confounding variables such as education, exercise, smoking, and alcohol consumption were used for the analysis and participants who had cardiac and renal disease were excluded in this study, preexisting diseases and types of treatment that may affect urinary sodium excretion could not be completely determined. In addition, there may be a problem in applying these results to other ethnic groups, because the participants of this study were limited to only Koreans.

Despite these limitations, our findings indicate that 24-hour urinary sodium calculated through Tanaka's equation using a spot urine specimen was associated with the prevalence of metabolic syndrome in Korean adults. This is the first study on the association between 24hour urinary sodium and metabolic syndrome using the KNHANES data that is representative of Koreans. Considering the clinical correlation between 24-hour urinary sodium and daily sodium intake, these findings may be meaningful evidence showing that sodium intake may be an important factor in the development of metabolic syndrome. Therefore, an interventional trial examining whether a low sodium diet will retard the development of metabolic syndrome is needed.

\section{CONFLICT OF INTEREST}

No potential conflict of interest relevant to this article was reported.

\section{REFERENCES}

1. Smith-Spangler CM, Juusola JL, Enns EA, Owens DK, Garber AM. Population strategies to decrease sodium intake and the burden of cardiovascular disease: a cost-effectiveness analysis. Ann Intern Med 2010;152:481-7, W170-3.

2. Vedovato M, Lepore G, Coracina A, Dodesini AR, Jori E, Tiengo A, et 
al. Effect of sodium intake on blood pressure and albuminuria in type 2 diabetic patients: the role of insulin resistance. Diabetologia 2004;47:300-3.

3. Larsen SC, Angquist L, Sorensen TI, Heitmann BL. 24h urinary sodium excretion and subsequent change in weight, waist circumference and body composition. PLoS One 2013;8:e69689.

4. Libuda L, Kersting M, Alexy U. Consumption of dietary salt measured by urinary sodium excretion and its association with body weight status in healthy children and adolescents. Public Health Nutr 2012;15:433-41.

5. Nishida C, Uauy R, Kumanyika S, Shetty P. The joint WHO/FAO expert consultation on diet, nutrition and the prevention of chronic diseases: process, product and policy implications. Public Health Nutr 2004;7:245-50.

6. Cogswell ME, Zhang Z, Carriquiry AL, Gunn JP, Kuklina EV, Saydah $\mathrm{SH}$, et al. Sodium and potassium intakes among US adults: NHANES 2003-2008. Am J Clin Nutr 2012;96:647-57.

7. Noh HM, Park SY, Lee HS, Oh HY, Paek YJ, Song HJ, et al. Association between high blood pressure and intakes of sodium and potassium among Korean adults: Korean National Health and Nutrition Examination Survey, 2007-2012. J Acad Nutr Diet 2015;115:1950-7.

8. Lee HS, Duffey KJ, Popkin BM. Sodium and potassium intake patterns and trends in South Korea. J Hum Hypertens 2013;27:298-303.

9. Grundy SM. Hypertriglyceridemia, insulin resistance, and the metabolic syndrome. Am J Cardiol 1999;83:25F-29F.

10. Grundy SM, Cleeman JI, Daniels SR, Donato KA, Eckel RH, Franklin BA, et al. Diagnosis and management of the metabolic syndrome: an American Heart Association/National Heart, Lung, and Blood Institute Scientific Statement. Circulation 2005;112:2735-52.

11. Lim S, Shin H, Song JH, Kwak SH, Kang SM, Yoon JW, et al. Increasing prevalence of metabolic syndrome in Korea: the Korean National Health and Nutrition Examination Survey for 1998-2007. Diabetes Care 2011;34:1323-8.

12. Ge Z, Guo X, Chen X, Tang J, Yan L, Ren J, et al. Association between 24 $\mathrm{h}$ urinary sodium and potassium excretion and the metabolic syndrome in Chinese adults: the Shandong and Ministry of Health Action on Salt and Hypertension (SMASH) study. Br J Nutr 2015;113:9961002.

13. Unal A, Kocyigit I, Sipahioglu MH, Tokgoz B, Oymak O. The relationship between daily urinary sodium excretion and metabolic syndrome in patients with kidney transplantation. Ann Transplant 2014;19:397402.

14. Baudrand R, Campino C, Carvajal CA, Olivieri O, Guidi G, Faccini G, et al. High sodium intake is associated with increased glucocorticoid production, insulin resistance and metabolic syndrome. Clin Endocrinol (Oxf) 2014;80:677-84.
15. Hoffmann IS, Cubeddu LX. Salt and the metabolic syndrome. Nutr Metab Cardiovasc Dis 2009;19:123-8.

16. Rhee MY, Kim JH, Kim YS, Chung JW, Bae JH, Nah DY, et al. High sodium intake in women with metabolic syndrome. Korean Circ J 2014;44:30-6.

17. Raisanen JP, Silaste ML, Kesaniemi YA, Ukkola O. Increased daily sodium intake is an independent dietary indicator of the metabolic syndrome in middle-aged subjects. Ann Med 2012;44:627-34.

18. Huh JH, Lim JS, Lee MY, Chung CH, Shin JY. Gender-specific association between urinary sodium excretion and body composition: analysis of the 2008-2010 Korean National Health and Nutrition Examination Surveys. Metabolism 2015;64:837-44.

19. McLean RM. Measuring population sodium intake: a review of methods. Nutrients 2014;6:4651-62.

20. Koo H, Lee SG, Kim JH. Evaluation of random urine sodium and potassium compensated by creatinine as possible alternative markers for 24 hours urinary sodium and potassium excretion. Ann Lab Med 2015;35:238-41.

21. Koo HS, Kim YC, Ahn SY, Oh SW, Kim S, Chin HJ, et al. Estimating 24hour urine sodium level with spot urine sodium and creatinine. J Korean Med Sci 2014;29 Suppl 2:S97-S102.

22. Grundy SM, Cleeman JI, Daniels SR, Donato KA, Eckel RH, Franklin BA, et al. Diagnosis and management of the metabolic syndrome: an American Heart Association/National Heart, Lung, and Blood Institute scientific statement. Curr Opin Cardiol 2006;21:1-6.

23. Yoon NH, Yoo S, Kim H, Han Y. Routine screening and consultation facilitate improvement of metabolic syndrome. J Korean Med Sci 2015;30:1092-100.

24. Lee SY, Park HS, Kim DJ, Han JH, Kim SM, Cho GJ, et al. Appropriate waist circumference cutoff points for central obesity in Korean adults. Diabetes Res Clin Pract 2007;75:72-80.

25. Gibson S. Salt intake is related to soft drink consumption in children and adolescents: a link to obesity? Hypertension 2008;51:e54.

26. Ma Y, He FJ, MacGregor GA. High salt intake: independent risk factor for obesity? Hypertension 2015;66:843-9.

27. Lee SK, Kim MK. Relationship of sodium intake with obesity among Korean children and adolescents: Korea National Health and Nutrition Examination Survey. Br J Nutr 2016;115:834-41.

28. Ando K, Fujita T. Pathophysiology of salt sensitivity hypertension. Ann Med 2012;44 Suppl 1:S119-26.

29. Lewicka S, Nowicki M, Vecsei P. Effect of sodium restriction on urinary excretion of cortisol and its metabolites in humans. Steroids 1998;63:401-5.

30. Fonseca-Alaniz MH, Brito LC, Borges-Silva CN, Takada J, Andreotti S, Lima FB. High dietary sodium intake increases white adipose tissue mass and plasma leptin in rats. Obesity (Silver Spring) 2007;15:2200-8. 